\title{
MÉXICO: ESTRUCTURA REFLEJANTE DE LA PERCEPCIÓN DE EFICIENCIA DE USO DE INTERNET
}

\author{
Cruz García Lirios \\ Universidad Nacional Autónoma de México - ENTS
}

http://dx.doi.org/10.5209/rev_NOMA.2012.41769

\begin{abstract}
Resumen: Se entrevistaron a 188 usuarios de una biblioteca pública de la Ciudad de México con el propósito de establecer la estructura bidimensional de sus capacidades percibidas de cibernavegación. Los resultados demuestran la verosimilitud de dos de tres hipótesis alternas. A partir del estado del arte, se construyó la definición de Autoeficiencia Electrónica para denotar la percepción de habilidades al momento de navegar en Internet. Se demostró la confiabilidad, validez de dos constructos, validez discriminante, estructura bidimensional y ajuste del modelo de medición en relación con el modelo estructural. A la luz de estos resultados, se expone la relevancia de la autoeficiencia electrónica en el Sistema Educativo Mexicano.
\end{abstract}

Palabras clave: sistema educativo mexicano, internet, autoeficacia, autoeficiencia

\section{Mexico: Reflective structure of the internet use self-efficacy}

\begin{abstract}
They were interviews to 188 users of a public library of the City of Mexico in order to establish the bidimensional structure of its perceived capacities of cyber navigation. The results demonstrate the probability of two of three alternating hypotheses. From the state-of-the-art, the definition of Electronic Self-Efficiency was constructed to denote the perception of abilities at the time of sailing in Internet. One demonstrated the trustworthiness, validity of two constructs, discriminating validity, bidimensional structure and fits of the model of measurement in relation to the structural model. In the light of these results, the relevance of the Electronic Self-Efficiency in the Mexican Educative System is exposed.
\end{abstract}

Keywords: mexican educative system, internet, self-efficacy, electronic self-efficiency.

La matrícula estudiantil de los sistemas educativos es su principal indicador de eficiencia. En el caso del Sistema Educativo Mexicano, los porcentajes de matriculación han sido incrementales y a partir de ellos se ha deducido un aumento eficiente. A medida que crece la cantidad de individuos registrados en el sistema educativo, se considera que existen altas probabilidades de aprovechamiento, competitividad y titulación. 


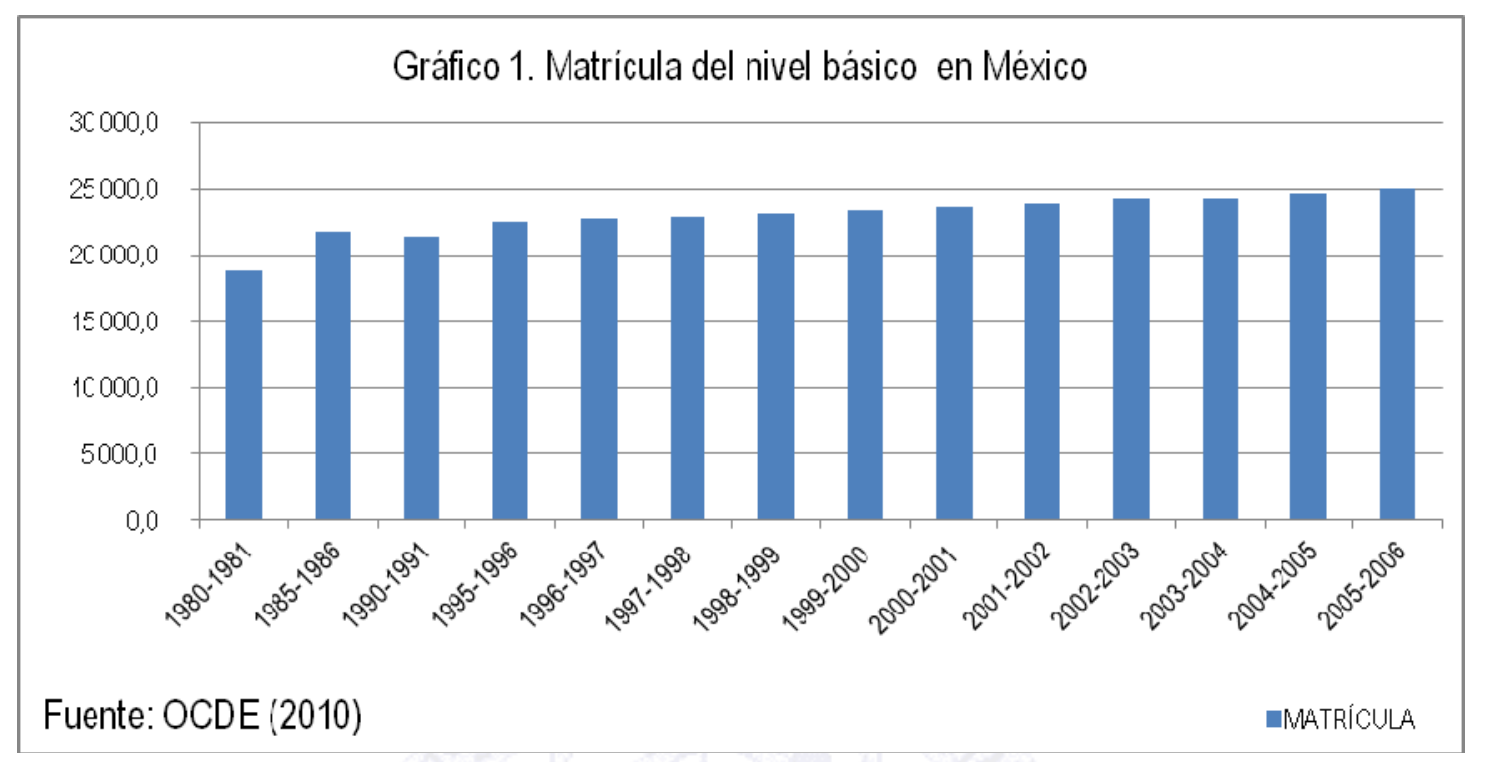

En el nivel básico, el sistema educativo registra un crecimiento significativo que inicia en 1980 cuando cerca de 18 mil estudiantes asistían a las aulas. Este crecimiento ha sido paulatino hasta alcanzar la cifra de 25 mil alumnos reconocidos por la Secretaria de Educación Pública (ver gráfico 1). No obstante, el aumento de la matrícula en el nivel básico no corresponde con los altibajos en las escuelas normalistas donde se instruyen a los futuros profesores de nivel básico.

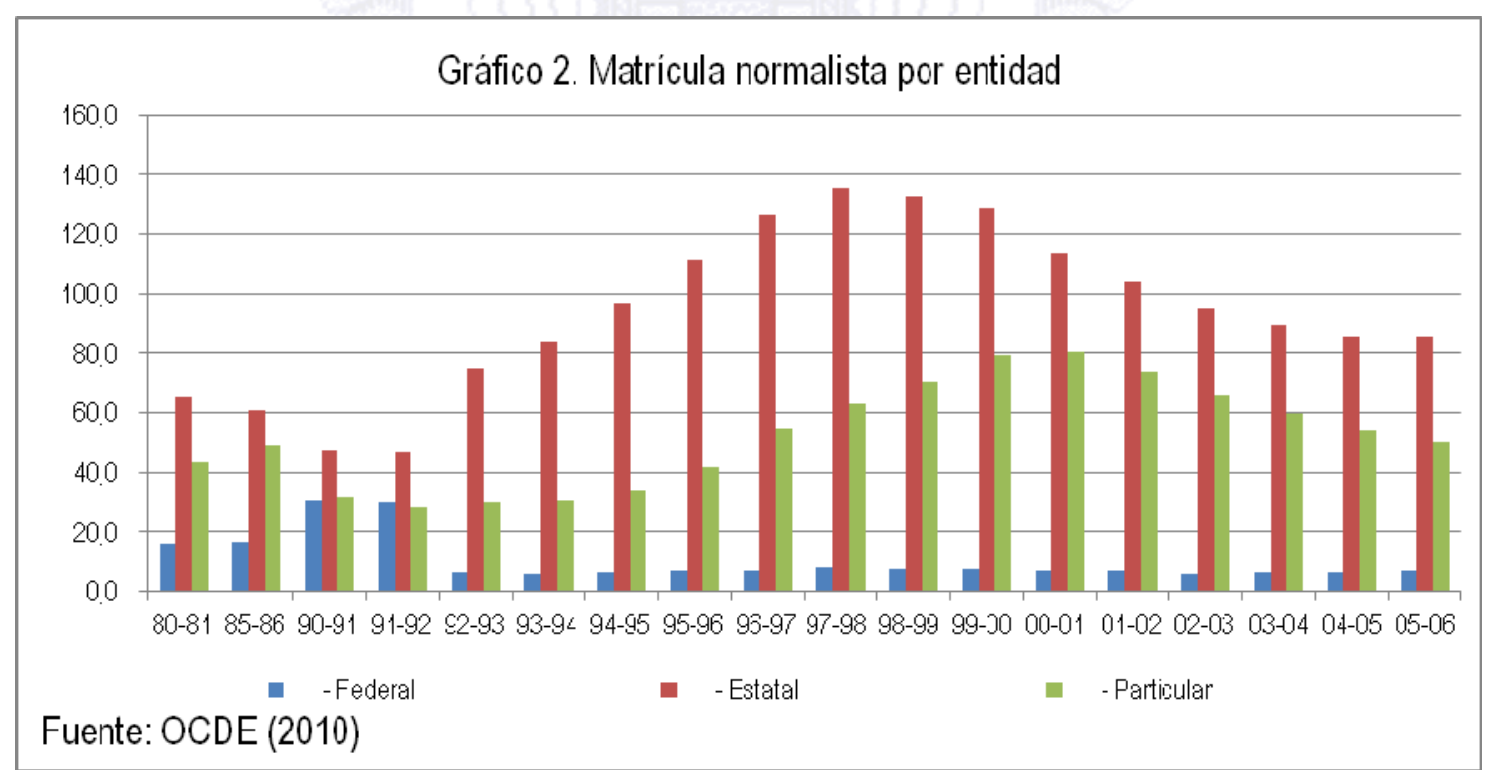

Durante el mismo periodo de 1980 a 2006 la matrícula normalista sólo ha incrementado en un 20 por ciento. Incluso, en el caso de las instituciones federales ha disminuido a partir de 1991 cuando alcanzó el 25 por ciento del total de la matrícula (ver gráfico 2). En contraste, la matrícula de universitarios y tecnólogos ha crecido intensi- 
ficadamente en el caso de las universidades y discretamente en el caso de los tecnológicos.

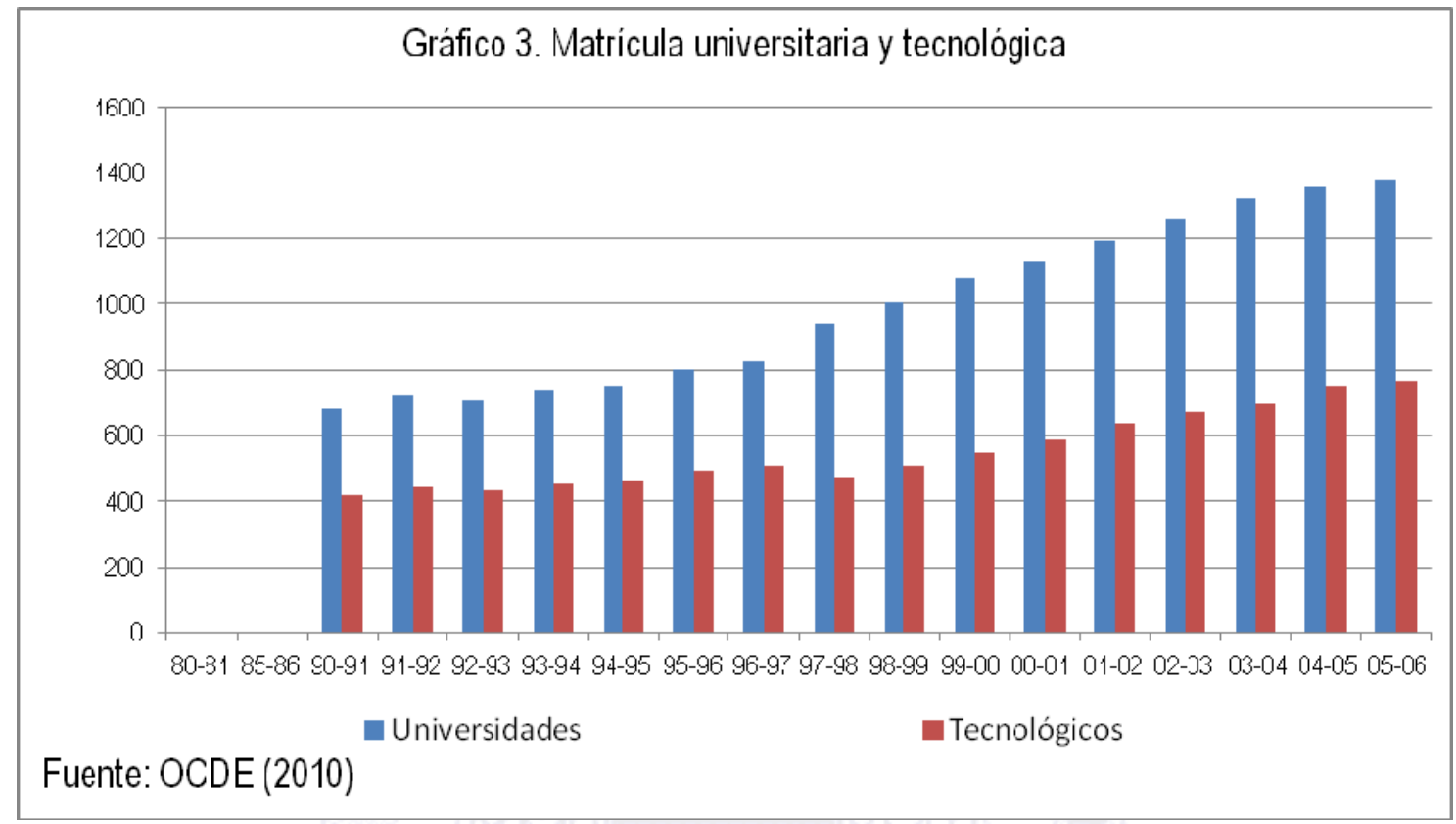

A partir de 1990 la matrícula universitaria ha crecido sustancialmente hasta alcanzar en el 2006 un total de un millón 377 mil estudiantes. En el caso de los institutos tecnológicos, éstos han visto crecer el número de sus estudiantes en 796 mil (ver gráfico 3).

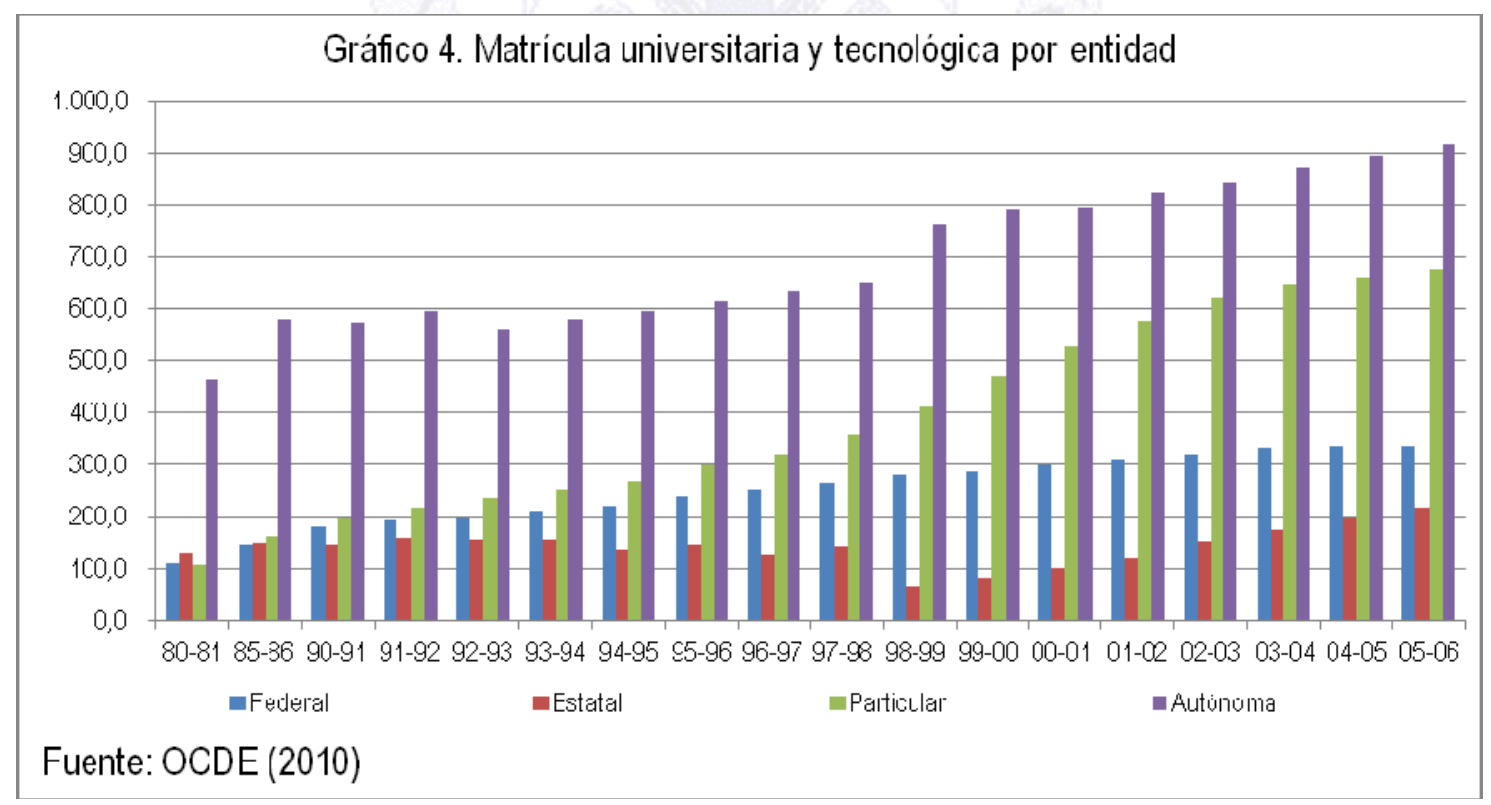


En definitiva, el crecimiento de la matrícula universitaria es evidentemente un proyecto de las universidades autónomas y de la iniciativa privada. Ambos han invertido sustancialmente hasta lograr un total de un millón 594 mil estudiantes en sus aulas (ver gráfico 4).

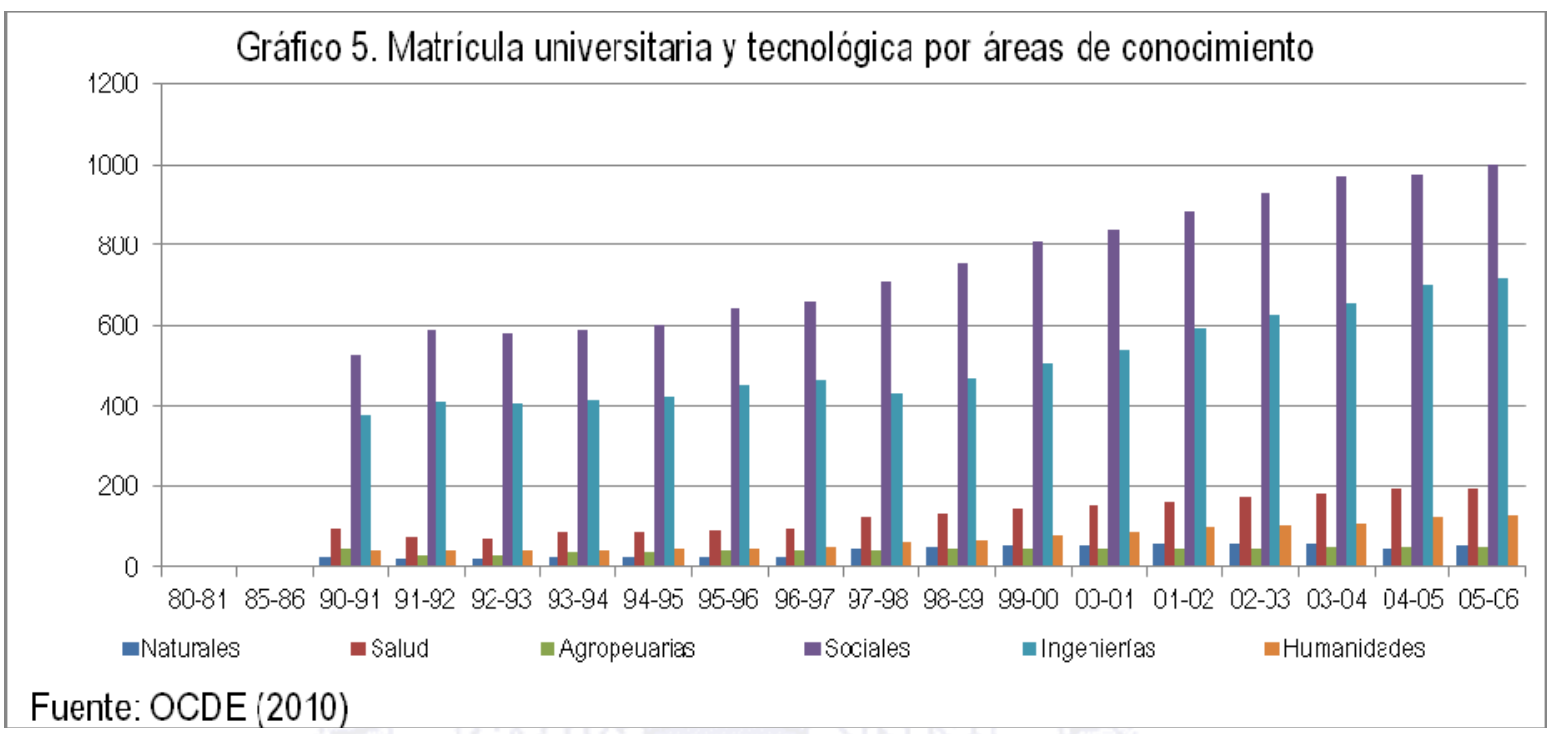

No obstante el crecimiento exponencial de la matrícula en el nivel superior, las inversiones parecen orientarse en formar científicos sociales más que profesionistas de la naturaleza, salud y tecnología. En efecto, en 2006 el sistema educativo mexicano formaba un millón de científicos sociales, 52 mil profesionistas de ciencias exactas y naturales, 195 estudiantes de la salud, 50 mil tecnólogos agropecuarios, 719 mil ingenieros y 128 mil humanistas (ver gráfico 5).

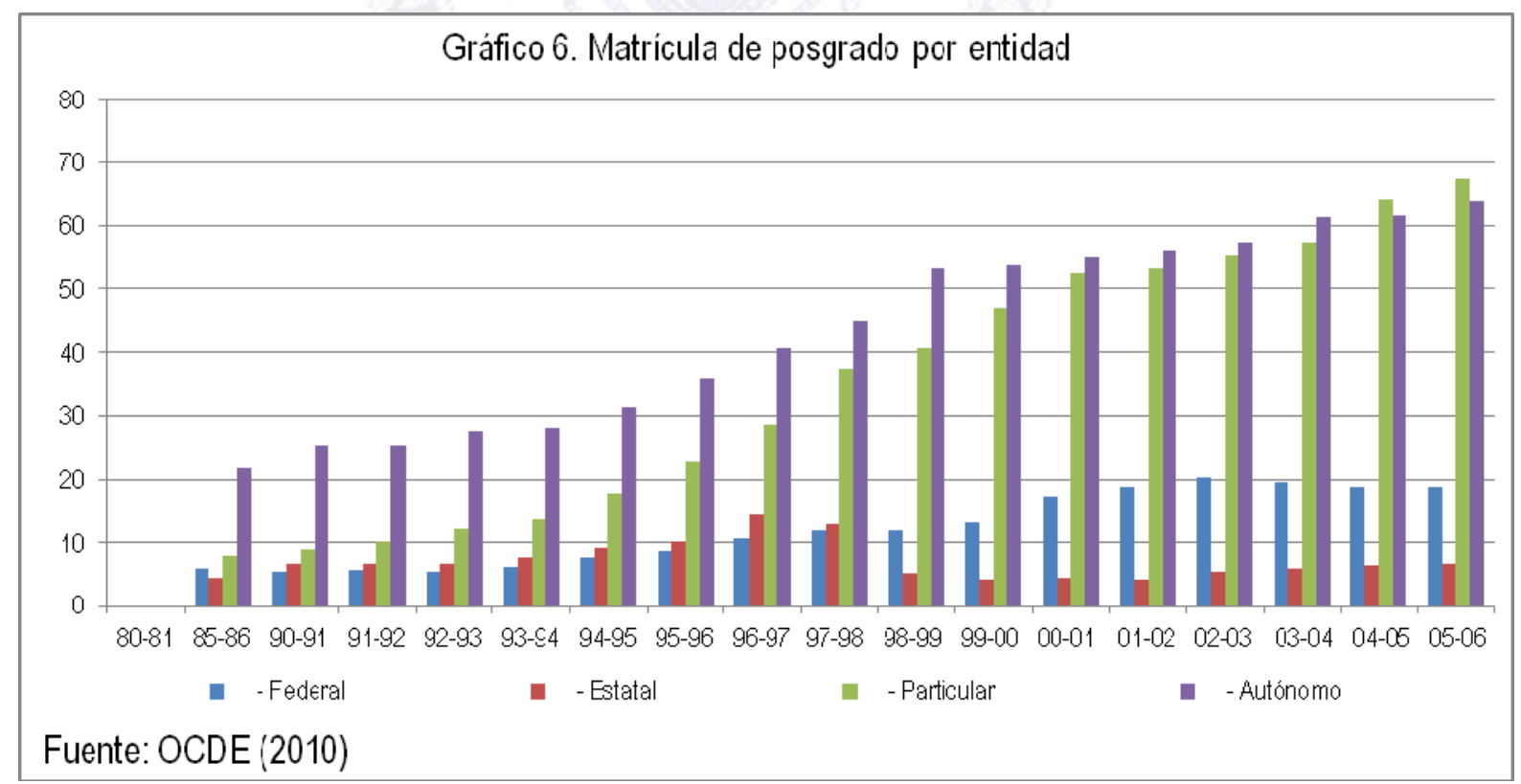


Respecto al nivel de posgrado, la tendencia es muy similar a la tendencia del nivel superior. Son las instituciones autónomas y los colegios particulares los que más invierten en la formación de especialistas, maestros y doctores. En el año 2006 la tendencia indica que las escuelas particulares superan a las universidades autónomas en la formación de posgraduados. Hasta el año 2004, las universidades autónoma superaban ligeramente a las particulares en el número de estudiantes, pero en el siguiente año, la iniciativa privada ha encontrado un mercado en el que esta dispuesto a invertir. Los 67 mil 448 estudiantes inscritos en el sistema educativo particular son una muestra de la oportunidad de negocios en el sector educativo nacional (ver gráfico 6).

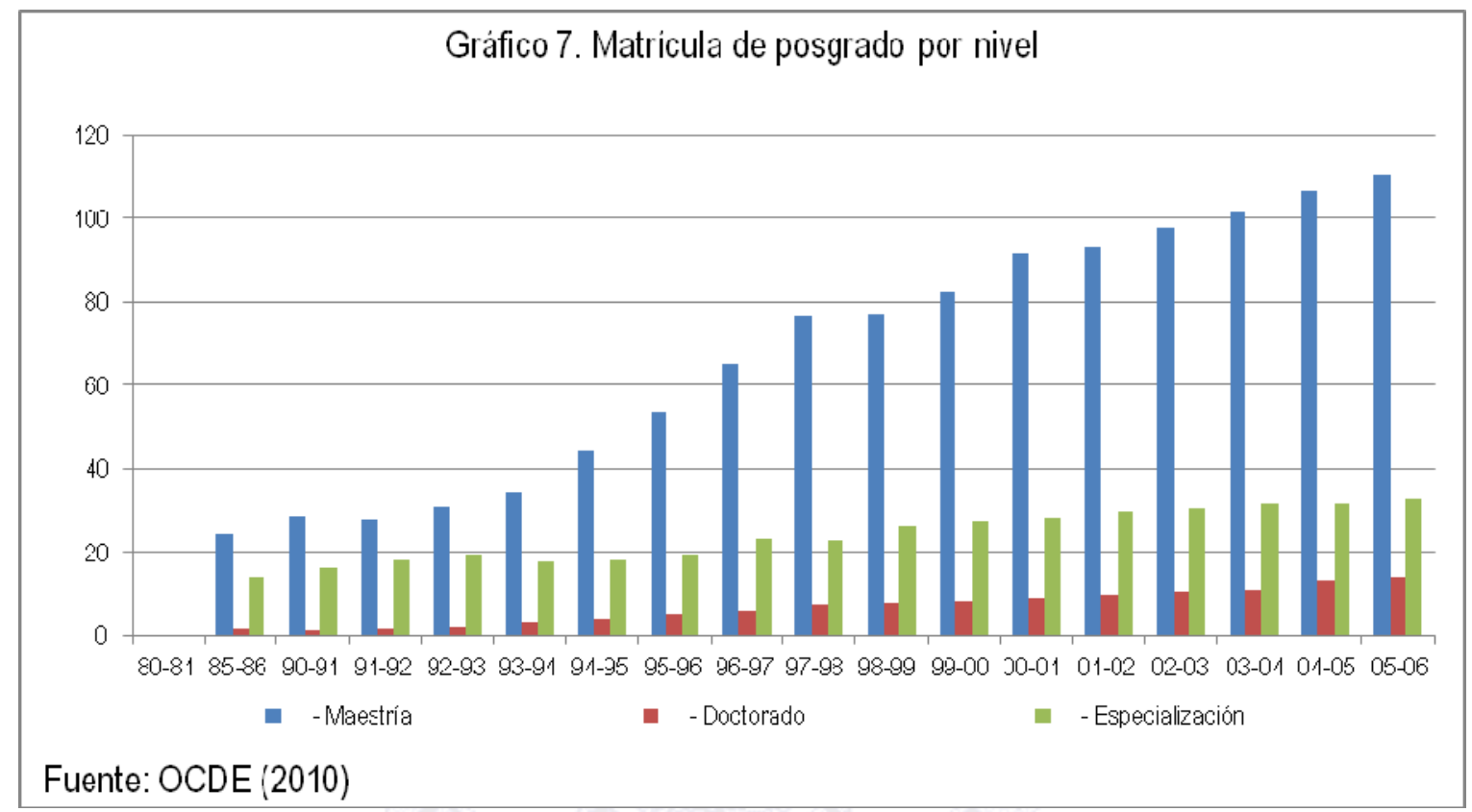

En el caso de las maestrías éstas han alcanzado un crecimiento sin precedentes. Durante el periodo de 1985 a 2006 la oferta de maestrías se ha incrementado cuatro veces más. En contraste, los programas de doctorado sólo cuanto con 13 mil 800 aspirantes al Sistema Nacional de Investigadores (ver gráfico 7).

En efecto, el crecimiento de la matrícula parece obedecer a una lógica de mercado enfocada en la formación de maestros con conocimientos administrativos jurídicos y a una política educativa austera en la formación de científicos investigadores. Una consecuencia de estas dos tendencias es el uso exponencial de Internet y sus correspondientes valores, percepciones, creencias, habilidades, motivos, actitudes, intenciones y competencias por parte de los estudiantes y profesionistas. 


\section{Autoeficacia percibida}

En la concepción de Bandura (1977; 1982; 1993; 1994; 1995; 2001) la autoeficacia es una percepción y/o una creencia motivada por ensayos de aciertos y errores personales o impersonales llevados a cabo deliberada o discursivamente. Dado que la autoeficacia alude al fracaso, pero principalmente al éxito, aún a pesar de aquellos ensayos fallidos que incitan al logro, la percepción y creencia de autoeficacia se sustenta en la consecución de objetivos esperados más que en la competitividad, el reconocimiento o el aprendizaje vicario. Si la autoeficacia es un sistema de percepciones y creencias enfocada al éxito, entonces el grupo al que pertenece o quiere pertenecer el agente autoeficaz esta relacionado con el éxito. Debido a que los grupos son diversos, la autoeficacia varía en función de esta diversidad. Un grupo competitivo atribuye éxito a uno de sus integrantes cuando éste ha sobrepasado los logros antecedentes, que por cierto estaban fijados por el grupo. En este sentido, el concepto de autoeficiencia parece fehacientemente ajustado a la influencia de un grupo sobre los objetivos, el sistema y logros de un individuo.

La autoeficiencia también sería un sistema de percepciones y creencias, pero a diferencia de la autoeficacia, éstas estarían orientadas a la ejecución normada de un procedimiento o tecnología. Los factores que impulsan la autoeficacia serían idénticos en el caso de la autoeficiencia. Si la competitividad, el reconocimiento y el aprendizaje vicario impulsan la autoeficacia, entonces la autoeficiencia también tendría ese impulso. No obstante, la autoeficiencia requiere de una motivación puntual: la percepción de facilidad de uso de la tecnología.

\section{Autoeficiencia computacional}

Los estudios de la autoeficiencia computacional han establecido relaciones asociativas y causales entre percepciones, creencias, actitud, experiencia, conocimiento, habilidades, confort, ansiedad, utilidad, afectividad, control y facilidad percibida.

Busch (1995) estableció la predicción de la autoeficacia computacional a partir de la experiencia previa. Este hallazgo concuerda con el estudio de Khorrami (2001) en el que demostró una asociación positiva y significativa entre las creencias de control y la disposición a teclear. Particularmente, Durndell y Haag (2002) sobre el aumento de la ansiedad al momento de manejar el ordenador. Baebite y Weiss (2004) predijeron el confort computacional y el ciber-confort a partir de la eficiencia computacional y los avances en la capacidad percibida. Hsu y Chiu (2004) demostraron el efecto exógeno de la ciber autoeficiencia mediante el ajuste de un modelo estructural. En dicha estructura, el uso de una tecnología esta determinado por el efecto de la ciber autoeficiencia a través de la intención y la actitud hacia la tecnología. Se trata de una estructura que explica un proceso deliberado, planificado y sistemático de adopción, aceptación y ciber-uso. Jonhson y Wardlow (2004) encontraron que la autoeficiencia asociada con la ansiedad en el uso computacional incrementaba la predicción y la significancia del uso de Internet. Sam, Othman y Nordin (2005) establecieron diferencias significativas entre las actitudes hacia Internet, la autoeficiecia computacional y la ciber-ansiedad con respecto a habilidades de almacenamiento de información. Chu (2006) al demostrar que la percepción de eficiencia variaba en función del uso 
previo de la computadora. La percepción de logro estaba determinada el número de ensayos en torno al manejo del computador. Torkzadeh, Chang y Demirhan (2006) demostraron diferencias individuales significativas entre hombres y mujeres que recibieron adiestramiento y registraban ansiedad y actitud hacia la ciber-navegación con respecto a la autoeficiencia computacional y la ciber-autoeficiencia. Wu y Tsai (2006) encontraron una asociación entre la efectividad y la autoeficiencia. No obstante, los estudios psicológicos han consolidado el efecto directo de la autoeficiencia sobre la percepción de utilidad. Bennet, Zimmer, Gundlach y Harrison (2008) encontraron, mediante un modelo estructural, la incidencia de la internalización y la externalización sobre la ciber-capacidad percibida. El ajuste de la estructura se demostró con cuatro muestras diferentes. No obstante, la covarianza entre ambos factores exógenos es cercana a la unidad lo cual limita su validez discriminante. Pareskova, Bouta y Papagianni (2008) demostraron que las habilidades básicas, los avances de las habilidades y el manejo de datos están asociados con la eficiencia computacional. Un incremento en el manejo de la computadora, los archivos y la captura de datos aumentaban la percepción de eficiencia. Reid y Levy (2008) demostraron, mediante un modelo de trayectorias, la predicción indirecta de la intención de uso a partir de la autoeficiencia computacional. En esta trayectoria de predicción, la percepción de utilidad, facilidad de uso percibida y actitud hacia la computación transmitieron significativamente el efecto de la ciber-capacidad percibida. Vekiri y Chonaki (2008) encontraron que la ansiedad, en el caso de las mujeres y la frecuencia de uso, en el caso de los hombres, están relacionados directa, positiva y significativamente con la autoeficiencia computacional. Saadé y Kira (2009) establecieron en un modelo mediacional el efecto directo e indirecto de la ciber ansiedad sobre la percepción de utilidad. Dicho efecto, también se incrementaba a través de la autoeficiencia computacional. Teh, Chong, Yong y Yew (2010) plantean que los logros configuran avances de autoeficiencia los cuales determinan el nivel de conocimiento. La relevancia de los avances en las habilidades básicas es otro determinante de la autoeficiencia computacional. En la medida en que las habilidades de manejo de archivos y datos se desarrollan propician un incremento en los conocimientos y las capacidades percibidas. Por su parte, Chuo, Tsai, Lan y Tsai (2011) demostraron la predicción de la intención de uso computacional a partir de la Cibereficiencia mediada por la percepción de utilidad y facilidad de uso percibida. Simsek (2011) encontró que un incremento en los valores de la capacidad percibida incide en un aumento de los valores intencionales regulados por los valores perceptuales. Se trata de un efecto indirecto en el que las creencias de eficiencia interactúan con la ansiedad.

¿Cuál es la estructura de la autoeficiencia electrónica? ¿Cuáles son las dimensiones de ésta estructura? ¿Cuál es el ajuste de ésta estructura? ¿Cuáles es el disturbio de ésta estructura?

\section{Método}

Sujetos. Se seleccionaron intencionalmente 188 usuarios de la biblioteca México. 141 mujeres (75 por ciento) y 47 hombres ( 25 por ciento). 62 tienen ingresos menores a 3000 pesos mensuales (33 por ciento), 79 entre 3000 y 6000 (42 por ciento) y 47 ganan más de 6000 al mes (25 por ciento). 55 tienen el grado de licenciatura 
(29,3 por ciento), 82 tienen el bachillerato ( 43,6 por ciento) y 51 tienen la secundaria (27,1 por ciento).

Variables. Autoeficiencia Electrónica es el grado de capacidad percibida en torno a la navegación en Internet. A diferencia de los conceptos de autoeficacia y autoeficiencia computacional, la autoeficiencia electrónica alude solamente a percepciones de capacidades o habilidades soslayando la experiencia vicaria y la motivación al logro.

Hipótesis. Debido a que el modelamiento de ecuaciones estructurales permite la demostración de varias hipótesis (Kline, 1998: 9), se plantearon seis; tres alternas y tres nulas.

Ho. La estructura hipotética de la relaciones reflejantes de la percepción de eficiencia de uso de internet se ajusta a la estructura estimada.

Ha: La estructura hipotética de la relaciones reflejantes de la percepción de eficiencia de uso de internet es diferente a la estructura estimada.

Ho: La estructura hipotética de la relaciones reflejantes de la percepción de eficiencia de uso de internet explica el mayor porcentaje de la varianza total.

Ha: La estructura hipotética de la relaciones reflejantes de la percepción de eficiencia de uso de internet explica el menor porcentaje de la varianza total.

Ho: La estructura hipotética de la relaciones reflejantes de la percepción de eficiencia de uso de internet esta influida por variables endógenas.

Ha: La estructura hipotética de la relaciones reflejantes de la percepción de eficiencia de uso de internet esta influida por variables exógenas.

Instrumento. Escala de Autoeficiencia Electrónica incluye 12 reactivos que miden la capacidad percibida en torno a la ciber-navegación con cuatro opciones de respuesta que van desde muy improbable hasta muy probable.

Procedimiento. Debido a que el perfil del usuario de internet es académico, se seleccionó a aquellos individuos que se encontraban en el vestíbulo de la biblioteca y se les solicito su participación en la contestación del cuestionario. Trascurridos diez minutos se les pidió el cuestionario y se revisaron las respuestas. En los casos en los que había una sola respuesta o la ausencia total o parcial de ellas, se les pidió que escribieran al reverso la razón por la que contestaron repetitivamente o en su caso, la ausencia de respuestas. Posteriormente, se capturan las respuestas en los programas estadísticos SPSS versión 17 y AMOS versión 6

\section{Resultados}

El proceso de modelamiento con ecuaciones estructurales requiere en primera instancia, la distribución normal de las respuestas a los rasgos de la autoeficiencia electrónica. El estadístico en el que se puede observar una distribución normal por no afectar la varianza de los datos es la curtosis (Kurtosis). Se trata del movimiento de la masa de datos hasta un punto tal en el que la distribución se considera normal. Un valor positivo indica un peso excesivo de las colas con un apuntalamiento necesario y un valor negativo un peso ligero con un achatamiento corres- 
pondiente. Ambas reflejan la normalidad en distribuciones unimodales y simétricas, pero la curtosis es diferente a la variabilidad de los datos (DeCarlo, 1997: p. 295). Puede observarse en la tabla 3 que la curtosis refleja una distribución normal. los valores positivos y negativos de la masa de kurtosis muestran una concentración de los valores en el centro al mismo tiempo que aligeran el peso de las colar y achatan la forma.

Tabla 1. Distribución normal de la Cibereficiencia

\begin{tabular}{|c|c|c|c|c|}
\hline Código & Reactivo & Media & Desviación & Curtosis \\
\hline Ce1 & $\begin{array}{l}\text { En la sección amarilla puedo eliminar las } \\
\text { ofertas inesperadas. }\end{array}$ & 2.46 & 1.020 & -1.094 \\
\hline $\mathrm{Ce} 2$ & $\begin{array}{l}\text { En google puedo evitar la publicidad eró- } \\
\text { tica repentina. }\end{array}$ & 2.84 & 1.159 & -1.305 \\
\hline Ce3 & $\begin{array}{l}\text { En wikipedia puedo elegir la información } \\
\text { científica que necesito. }\end{array}$ & 2.44 & .937 & -.879 \\
\hline Ce4 & $\begin{array}{l}\text { En amazon puedo seleccionar las ofertas } \\
\text { que necesito. }\end{array}$ & 2.38 & 1.161 & -1.454 \\
\hline Ce5 & $\begin{array}{l}\text { En myspace puedo elegir con quien cha- } \\
\text { tear. }\end{array}$ & 2.47 & 1.139 & -1.411 \\
\hline Ce6 & $\begin{array}{l}\text { En google puedo inscribirme a los cursos } \\
\text { que necesito. }\end{array}$ & 2.22 & 1.114 & -1.149 \\
\hline $\mathrm{Ce} 7$ & $\begin{array}{l}\text { En amazon puedo comprar los productos } \\
\text { que busco. }\end{array}$ & 2.18 & 1.044 & -1.073 \\
\hline Ce8 & $\begin{array}{l}\text { En el aviso oportuno puedo contratar los } \\
\text { servicios que me gusten. }\end{array}$ & 2.70 & 1.178 & -1.412 \\
\hline Ce9 & $\begin{array}{l}\text { En facebook puedo chatear con las co- } \\
\text { munidades científicas que busco. }\end{array}$ & 2.48 & 1.062 & -1.214 \\
\hline Ce10 & $\begin{array}{l}\text { En amazon puedo encontrar ofertas es- } \\
\text { peciales. }\end{array}$ & 2.34 & 1.133 & -1.412 \\
\hline Ce11 & $\begin{array}{l}\text { En youtube puedo bajar imágenes eróti- } \\
\text { cas especiales. }\end{array}$ & 2.61 & 1.172 & -1.456 \\
\hline Ce12 & $\begin{array}{l}\text { En google puedo comunicarme con los } \\
\text { científicos que busco. }\end{array}$ & 2.51 & 1.111 & -1.343 \\
\hline
\end{tabular}

Demostrada la distribución normal se procedió a establecer la validez de la autoeficiencia electrónica. A decir de Kline (1996: p. 10) los indicadores pueden configurar constructos que representen una diversidad de procesos psicológicos latentes y con ello mejorar una consistencia interna imperfecta. También señala que las variables latentes pueden no estar definidas porque los indicadores por si solos son suficientes 
para inferir un factor común a todos ellos. La tabla 2 muestra la convergencia de indicadores en la autoeficiencia electrónica. Los valores de los indicadores cercanos a la unidad implican la validez de constructo y los valores cercanos al cero para otro constructo son muestra de validez discriminante.

Tabla 2. Validez de la Cibereficiencia

Código Reactivo

Cibereficiencia

Ce11 En youtube puedo bajar imágenes eróticas especiales.

$-.041$

Ce6 En google puedo inscribirme a los cursos que necesito.

Ce8 En el aviso oportuno puedo contratar los servicios que me gusten.

Ce10 En amazon puedo encontrar ofertas especiales.

Ce5 En myspace puedo elegir con quien chatear.

Ce3 En wikipedia puedo elegir la información científica que necesito.

Ce4 En amazon puedo seleccionar las ofertas que necesito.

Ce7 En amazon puedo comprar los productos que busco.

Ce12 En google puedo comunicarme con los científicos que busco.

Ce2 En google puedo evitar la publicidad erótica repentina.

Ce9 En facebook puedo chatear con las comunidades científicas que busco.

Ce1 En la sección amarilla puedo eliminar las ofertas inesperadas.

Kerlinger y Lee (2002: p. 415) definen a la confiabilidad en términos del incremento de varianza sistemática y la disminución del error aleatorio en relación con la varianza total. Si la varianza sistemática perfecta es equivalente a la unidad, entonces la varianza de error es la diferencia entre la varianza total y la varianza sistemática. La confiabilidad de la Cibereficiencia fue de .610 la cual se interpreta como suficientemente adecuada con una variabilidad sistemática cercana a la unidad. Si se restan estos valores a la unidad se obtienen valores de error cercanos al cero. Es decir, el instrumento que mide la autoeficiencia electrónica tiene una varianza sistemática óptima al mismo tiempo que es capaz de reducir el error si se mide $n$ veces la autoeficiencia con la misma muestra y el mismo instrumento. Kline (1998: p. 10) define a la covarianza como una correlación que puede ser estandarizada (media $=0$ y desviación $=1$ ) cuando sobrepasa los límites de la unidad negativa o positiva. También 
puede ser una correlación no estandarizada cuando los valores oscilan entre el rango de -1 a +1 . En las covarianzas de la tabla 3, los indicadores están asociados no estandarizadamente porque sus valores son menores a la unidad. Sólo en las correlaciones entre los indicadores de la Autorganización electrónica pueden apreciarse valores superiores a la unidad. En todas y cada una de las correlaciones, se observan valores positivos que se interpretan como el crecimiento de una variable que corresponde con el aumento de otra. Esto mismo sucede con el error de medición que es constante en cada indicador y se interpreta como una homocedasticidad óptima de relaciones lineales para los casos en que el valor de covarianza se aleja de cero.

Tabla 3. Las covarianzas entre los indicadores de la Cibereficiencia

\begin{tabular}{llllll}
\hline & CE12 & CE9 & CE2 & CE1 \\
\hline $\begin{array}{l}\text { En google puedo comunicarme con los científicos que } \\
\text { busco. }\end{array}$ & 1.229 & & & \\
$\begin{array}{l}\text { En facebook puedo chatear con las comunidades } \\
\text { científicas que busco. }\end{array}$ & .290 & 1.122 & & \\
$\begin{array}{l}\text { En google puedo evitar la publicidad erótica repentina. } \\
\begin{array}{l}\text { En la sección amarilla puedo eliminar las ofertas ines- } \\
\text { peradas. }\end{array}\end{array}$ & .400 & .459 & .627 & .749 & 1.035 \\
\hline
\end{tabular}

Kline (1998: p. 48) señala que una vez especificado el modelo (elaboración de relaciones hipotéticas entre indicadores y constructos) se procede a identificar el modelo (en el caso en que el programa pueda determinar la estructura teóricamente supuesta). En el presente estudio se seleccionó el parámetro chi cuadrada para probar la hipótesis de ajuste y en su caso re-especificar el modelo. El estadístico tiene una significancia que demuestra la aceptación de la primera hipótesis alterna. No obstante, Kline (2006) advierte que el parámetro chi cuadrada al ser sensible al tamaño de la muestra, debe ser corroborado con otros índices de ajuste. Estos parámetros demuestran la verosimilitud de una hipótesis cuando sus valores son cercanos a la unidad. En contraste, si sus valores tienden al cero, el investigador deberá aceptar la hipótesis nula. La tabla 4 muestra los valores de índices de ajuste cuyos valores son próximos a la unidad.. 
Modelo 1. Estructura de la Cibereficiencia

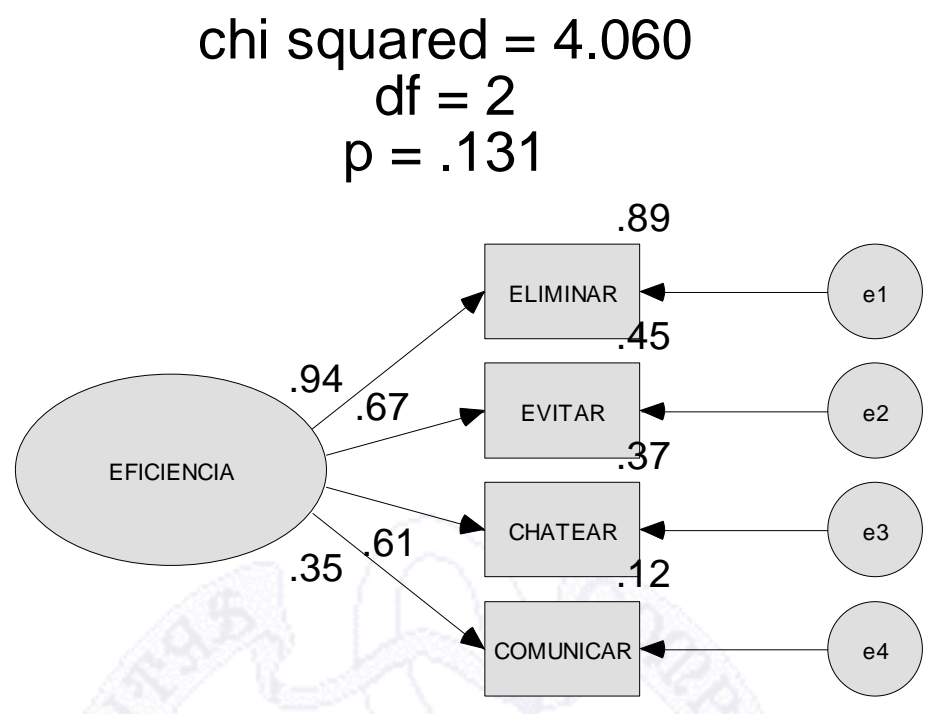

Tabla 4. Los ajustes y residuales de la estructura cibereficiente

$\begin{array}{llllllll}\text { GFI AGFI NFI } & \text { RFI } & \text { IFI } & \text { TLI } & \text { CFI } & \text { RMSEA } & \text { RMR }\end{array}$

\begin{tabular}{ll|l|l|l|l|l|l|lll} 
Estructura ciberefi- & .990 & .949 & .980 & .939 & .990 & .968 & .989 & .074 & .036
\end{tabular} ciente

En síntesis, se demostró una hipótesis alterna en torno al ajuste del modelo de medición a la estructura observada de la autoeficiencia electrónica. Sin embargo, el parámetro de disturbio en ambos factores muestra la incidencia de indicadores y variables latentes. Los estadísticos que miden el disturbio sugieren una estructura multidimensional de la autoeficiencia electrónica.

\section{Conclusión}

Al ser Internet un escenario de interrelación de múltiples personalidades tiende, en el caso de la autoeficiencia, a poseer dimensiones de compra-venta, enseñanzaaprendizaje, persuasión-disuasión, resistencia-cambio, conflicto-conciliación o competencia-solidaridad en las que las capacidades percibidas diversifican sus diseños, contenidos y funciones.

Se trata de una autoeficiencia en la que los ensayos se multiplican y con ello diversifican la noción de éxito o fracaso, el aprendizaje vicario o la competitividad. Un estudiante que navega por internet desarrolla un sistema de percepciones y creencias que provocan un crecimiento exponencial de su motivación al logro, comparación social o personal y sus capacidades-habilidades de consumo. 
No obstante esta diversidad, multiplicidad, polivalencia y heterogeneidad de la red, sus usuarios y anunciantes. El presente estudio demostró la bidimensionalidad de la autoeficiencia electrónica en una muestra de estudiantes que acuden regularmente a una biblioteca pública, pero que ello no impide que sus habilidades-capacidades puedan representar una tendencia de aceptación, uso y transformación de Internet.

La auto-organización y el auto-emprendimiento son factores que contribuyen a la heterogeneidad de la percepción de habilidades de ciber-navegación. El individuo que se percibe como una persona con habilidades deliberadas, planificadas y sistemáticas de navegación incrementa sus búsquedas de información y consumo en la red.

Sin embargo, el estado del arte reporta otros factores que potencializan la autoeficiencia electrónica. Tales son los casos de los valores, actitudes, intenciones y comportamientos que pueden ser parte de un sistema de reproducción y producción de competencias que se activan o modifican según las circunstancias de navegación en Internet.

Incluso, el estado del arte también advierte la diversidad de las capacidades percibidas al demostrar las relaciones asociativas y causales entre percepciones de riesgo, control, confort, utilidad y facilidad de uso de la web.

En tal sentido, se recomienda la exploración, medición y predicción de los factores mencionados en una estructura integral en la que la autoeficiencia electrónica sea especificada en un modelo de medición y demostrada en un modelo estructural. Los parámetros empleados en el presente estudio también demostrarían las hipótesis de multidimensionalidad, ajuste y disturbio.

En relación con el Sistema Educativo Mexicano la autoeficiencia electrónica advierte sobre las oportunidades y posibilidades de desarrollo educativo que necesariamente van acompañadas de la diversidad de capacidades percibidas por los usuarios de Internet al momento de navegar con objetivos educativos-académicos de enseñanza-aprendiza. Una diversificación de la autoeficiencia electrónica implicaría una heterogeneidad de planes y programas de estudio no reducibles a políticas de desarrollo económico tecnológico, sino complementarias de la libertad de ciber-expresión.

Ante la lógica de mercado y política económica tecnológica, la autoeficiencia electrónica se erige como una muestra de la multiculturalidad de México. Mientras el Sistema Educativo Mexicano parece crecer indiscriminadamente, la ciber-autoeficiencia estudiantil parece diversificarse. Mientras la matrícula aumenta sin límites, los usuarios de internet parecen multiplicar sus habilidades de ciber-navegación que les permiten, en un momento dado, quebrantar las formas de enseñanza-aprendizaje establecidas por autorganización y autoemprendimiento en torno al desarrollo personal, grupal y social que les permita amplificar la información, enriquecer el lenguaje, diversificar la cultura, multiplicar el consumo y heterogeneizar las redes sociales. 


\section{Referencias}

Bandura, A. (1977). Self-efficacy: toward a unifying theory of behavioral change. Psychological Review. 84, 191-215

Bandura, A. (1982). Self-efficacy. Mechanism in human agency. American Psychologist. 37, 122-147

Bandura, A. (1993). Perceived self-efficacy in cognitive development and functioning. Educational Psychologist. 28, 117-148

Bandura, A. (1994). Self-efficacy. In V. S. Ramachaudran (ed.). Encyclopedia and Human Behavior. (pp. 71-88). New York: Academic Press

Bandura, A. (1995). Exercise of personal and collective efficacy in changing societies. In A. Bandura (ed.). Self-efficacy in changing societies. (pp. 1-45). New York: Cambriedge University Press.

Bandura, A. (2001). Social cognitive theory: an agentic perspective. Annual Review of Psychology. 52, 1-26

Barbeite, F. \& Weiss, E. (2004). Computer self "efficacy and anxiety scales for a internet sample: testing measurement equivalence of existing measures and development of new scales. Computers in Human Behaviors. 20, 1-15

Bennett, J., Zimmer, C., Gundlach, M. \& McKnight, H. (2008). Internal and external dimensions of computer self-efficacy: an empirical examination. Transactions on Engineering Management. 55, 628-644

Busch, T. (1995). Geneder differences in self-efficacy and attitudes towards computers Journal of Educational Computers Research. 12, 147-158

Chu, Y. (2006). Levels of computer self efficacy , computer use and earnings in China. Economics Letters. 90, 427-432

Chuo, Y-H., Tsai, C-H., Lan, Y-L. \& Tsai, C-S. (2011). The effect of organizational support, self efficacy and computer anxiety on the usage intention of e-learning system in hospital. African Journal of Business Management. 5, 5518-5523

Compeau, D. \& Higgins, C. (1995). Computer self efficacy: development of measure and initial test. Mis Quarterly. 19, 189-211

DeCarlo, L. (1997). On the meaning and use of kurtosis. Psychological Methods. 2, 292-307

Durndell, A. \& Haag, Z. (2002). Computer self-efficacy, computer anxiety, attitudes toward internet and reported experience with the internet, by gender, in an East European sample. Computers in Human Behavior. 18, 521-535

Hsu, M-H. \& Chiu, C-M. (2004). Internet self efficacy and electronic service acceptance. Decision Support Systems. 38, 369-381

Jonhson, D. \& Warldlow, J. (2004). Computers experiences, self efficacy and knowledge of undergraduate student entering a land grant college of agriculture by year and gender. Journal of Agricultural Education. 45, 53-64 
Kerlinger, F. y Lee, H. (2002). Investigación del comportamiento. Métodos de investigación en Ciencias Sociales. México: McGraw Hill.

Khorrami, O. (2001). Researching computer self-efficacy. International Educational Journal. 2, 17-26

Kline, R. (1998). Principles and practice of structural equations modelling. New York: Guilford Press.

Kline, R. (2006). Reverse arrow dynamics. Formative measurement and feedback loops. In G. Hancock \& R. Mueller (coord.). Structural Equation Modeling: A Second Course. (pp. 43-68). Greenwood, CT: Information Age Publishing, Inc.

Liang, J.-C., \& Tsai, C.-C. (2008). Internet self-efficacy and preferences toward constructivist Internet-based learning environments: A study of pre-school teachers in Taiwan. Educational Technology \& Society, 11 (1), 226-237.

Paraskeva, F., Bouta, H. \& Papagianni, A. (2008). Individual characteristics and computer self-efficacy in secondary education teachers to integrated technology in education practice. Computers \& Education. 50, 1084-1091

Reid, M. \& Levy, Y. (2008). Integrating trust and computer self efficacy with TAM: an empirical assessment of customer acceptance of Banking Information System. Journal of Internet Banking and Commerce. 12, 1-18

Saadé, R. \& Kira, D. (2009). Computer anxiety in e-learning: the effect of computer self-efficacy. Journal of Information Technology Education. 8, 1-15

Sam, H. K., Othman, A. E. A., \& Nordin, Z. S. (2005). Computer Self-Efficacy, Computer Anxiety, and Attitudes toward the Internet: A Study among Undergraduates in Unimas. Educational Technology \& Society, 8 (4), 205-219.

Simsek, A. (2011). The relationships between computer anxiety and computer self efficacy. Contemporary Educational Technology. 2, 177-187

Teh, P-L., Chong, C-W., Yong, C-C. \& Yew, S-Y. (2010). Internet self-efficacy, computer self-efficacy, and cultural factor on knowledge sharing behavior. African Journal of Business Management. 4, 4086-4095

Torkzadeh, G., Cha, J. \& Demirhan, D. (2006). A contingency model of computer and internet self efficacy. Information \& Management. 43, 541-550

Vekiri, I. \& Chronaki, A. (2008). Gender issues in technology use: perceived social support, computer self-efficacy and value beliefs, and computer beyond school. Computers \& Education. 51, 1392-1404

Wu, Y-T. \& Tsai, C-C. (2006). University students internet attitudes and internet self efficacy: a study at tree universities in Taiwan. Cyberpsychology \& Behavior. 9, 441452 